UPR-0510T

\title{
GRACEFUL EXIT IN EXTENDED INFLATION AND IMPLICATIONS FOR DENSITY PERTURBATIONS
}

\author{
Robert Crittenden and Paul J. Steinhardt \\ Department of Physics \\ University of Pennsylvania
}

Philadelphia, PA 19104

\begin{abstract}
Two qualitatively different modes of ending superluminal expansion are possible in extended inflation. One mode, different from the one envoked in most extended models to date, easily avoids making big bubbles that distort the cosmic microwave background radiation (CMBR). In this mode, the spectrum of density fluctuations is found to be scale-free, $P(k) \propto k^{n}$, where $n$ might lie anywhere between 0.5 and 1.0 (whereas, previously, it appeared that the range $1.0>n \gtrsim 0.84$ was disallowed).
\end{abstract}


Extended inflation ${ }^{1,2}$ was introduced to resolve the fine-tuning problem that plagued previous inflationary models. As in "old inflation," ${ }^{3}$ the scale factor $a(t)$ begins to expand superluminally $(a(t)>t)$ when the universe supercools into a metastable false vacuum phase during a strongly first order phase transition. Unlike old inflation, though, graceful exit can be achieved by bubble nucleation, quantum tunneling through the energy barrier. The added element is modifications to Einstein gravity that slow the expansion from exponential $(a(t)=\exp (H t)$ and constant Hubble parameter $H)$ to polynomial $(a(t)>t$, but $H$ steadily decreasing). For slowed expansion, the dimensionless parameter $\epsilon \equiv \Gamma / H^{4}$ — which measures the competition between the nucleation rate $\Gamma$ and the expansion rate - steadily increases. ${ }^{1}$ Initially, $\epsilon \ll 1$ and inflation dominates nucleation. In old inflation, $\epsilon$ is constant so inflation never stops. In extended inflation, $\epsilon$ steadily increases until $\epsilon>1$, at which point nucleation dominates expansion and the transition to the true vacuum is completed.

The purpose of this paper is to show that extended inflation offers two qualitatively distinct modes of "graceful exit." In the mode considered by most authors, inflation stops when bubbles of true vacuum consume the false vacuum. This mode can create problems of its own, including unacceptable inhomogeneities created by big bubbles. ${ }^{4,5}$ Our point here is to emphasize a second mode in which inflation ends while the universe is still trapped in the false phase. We argue that this mode automatically avoids the big bubble problem. We also examine the implications for the the density perturbation spectrum. In a spectrum characterized by a power-law, $P(k) \propto k^{n}$, scale invariance (a Harrison-Zel'dovich spectrum) corresponds to $n=1$. Previously, avoiding the big bubble problem seemed to require $n<0.84$, and, hence, a 
spectrum tilted away from strict scale-invariance. However, with the novel graceful exit mechanism, the range opens up to $1.0 \geq n \gtrsim 0.5$, including $n$ arbitrarily close to unity.

For the purposes of illustration, we will consider modified gravity theories in which a scalar field $\phi$ is non-minimally coupled to the scalar curvature, $\mathcal{R}$ :

$$
\mathcal{L}=-f(\phi) \mathcal{R}-\frac{1}{2}\left(\partial_{\mu} \phi\right)^{2}-\frac{1}{2}\left(\partial_{\mu} \sigma\right)^{2}-V(\sigma)+\mathcal{L}_{\text {other }}
$$

The field $\sigma$ is an order parameter (e.g., a Higgs field) for a strongly first order transition; during supercooling, $\sigma$ is trapped in a false vacuum phase with energy density is $V(\sigma)=M_{F}^{4}$. $\mathcal{L}_{\text {other }}$ includes all other matter fields and any potential for $\phi$. It is useful to recast the action in terms of a new scalar field, $\Phi \equiv f(\phi)$ and a function $\omega(\phi) \equiv \frac{f(\phi)}{2\left[f^{\prime}(\phi)\right]^{2}}$ :

$$
\mathcal{L}=-\Phi \mathcal{R}-\omega(\Phi) \frac{\left(\partial_{\mu} \Phi\right)^{2}}{\Phi}-\frac{1}{2}\left(\partial_{\mu} \sigma\right)^{2}-V(\sigma)+\mathcal{L}_{\text {other }}
$$

The original Brans-Dicke model corresponds to $f(\phi)=\xi \phi^{2}$, a special case in which $\omega(\Phi)=1 / 8 \xi$ is constant. ${ }^{1,6}$ The generic case is where $\omega$ is $\Phi$ dependent, ${ }^{2}$ a generalization which leads to two distinct modes of ending extended inflation:

Mode 1: Inflation ends by true vacuum bubbles consuming the false vacuum. This mode, considered in the first extended inflation papers, ${ }^{1,2,4,5,7}$ is exemplified by the original Brans-Dicke theory with constant $\omega$. While the universe is trapped in the false vacuum, the scale factor increases as a power-law, ${ }^{1}$ $a(t) \propto t^{\omega+\frac{1}{2}}$, and the Hubble parameter, $H=\dot{a} / a=\left(\omega+\frac{1}{2}\right) / t$, decreases uniformly. Hence, $\epsilon=\Gamma / H^{4}$ increases steadily, as desired.

Density perturbations are generated during inflation from two sources. 
Quantum fluctuations in $\phi$ result in a nearly scale-invariant spectrum: ${ }^{7,8}$

$$
\left.\frac{\delta \rho}{\rho}\right|_{H}(\lambda) \approx \frac{M_{F}^{2}}{m_{P}^{2}} g(\omega) \lambda^{4 /(2 \omega-1)}
$$

where $\left.\frac{\delta \rho}{\rho}\right|_{H}(\lambda)$ is the perturbation amplitude as length $\lambda$ re-enters the horizon after inflation; $m_{P}^{2} \equiv f(\phi)$ is the effective Planck mass at the end of superluminal expansion; and $g(\omega)$ is an $\omega$-dependent correction ${ }^{8}$ that is close to unity except for $\omega \rightarrow 1$. Note that a $\left.\frac{\delta \rho}{\rho}\right|_{H}$ spectrum which scales as $\lambda^{m}$ converts to a power spectrum $P(k) \propto k^{1-2 m}$. For $M_{F} / m_{P} \approx 10^{-(3-5)}$, but with no fine-tuning of couplings, $\left.\frac{\delta \rho}{\rho}\right|_{H}(\lambda)$ is consistent with recent COBE observations. A second source of inhomogeneities comes from big bubbles. ${ }^{4,5}$ If inflation continues until bubbles consume all remaining false vacuum, the distribution of bubble sizes at the end of inflation is $F\left(x>x_{0}\right) \approx \frac{1}{\left(1+H_{e} x_{0}\right)^{4 / \omega}}$, where $F\left(x>x_{0}\right)$ is the fractional volume occupied by bubbles of proper radius greater than $x_{0}$ and $H_{e}$ is the Hubble parameter at the end of inflation. ${ }^{5}$ To avoid distortion of the CMBR, we require $F\left(x>x_{0}\right)$ to be less than $10^{-4}$ for bubbles of supercluster scale or larger, $H_{e} x_{0} \geq 10^{25}$; this implies $\omega<25$.

The serious criticism of this mode is the disappointingly limited range of workable models. Although the phase transition can be completed for any $\omega<\infty$, only $\omega<25$ leads to acceptable inhomogeneities. (Observational constraints on Brans-Dicke theory conflict with $\omega<25$ if $\phi$ is massless, but are avoided by assuming a finite mass $\gtrsim 10^{-12} \mathrm{GeV}^{9}$ )

Mode 2: Inflation ends while the universe remains trapped in the false phase. Generically, $\omega$ is $\phi$-dependent and time-dependent, enabling a new mechanism for terminating superluminal expansion. For a very wide range of polynomial or exponential $f(\phi), \omega$ decreases sharply with increasing $\phi$. See Figure 1. When $\phi$ is small, $\omega>>1$ and the universe expands superluminally. Through the non-minimally coupling term, the false vacuum energy density 
drives $\phi$ to steadily increase. As $\phi$ grows, $\omega$ decreases and $a(t)$ accelerates less rapidly. The boundary, $\omega=1 / 2$, is critically important. Recall that the scale factor grows as $a(t) \approx t^{\omega+\frac{1}{2}}$, for constant $\omega .^{1}$ The expansion becomes subluminal — non-inflationary — once $\omega$ drops below $1 / 2$, even though the universe remains trapped in the false vacuum. Even after this point, $\phi$ continues to grow, the expansion rate continues to decrease, and the bubble nucleation parameter, $\epsilon$, continues to increase. Some time after inflation has stopped, $\epsilon$ finally increases above unity and bubble nucleation ends the transition to the false phase. As in Mode 1, several mechanisms ${ }^{5}$ are possible which can later pin $\phi$ at a fixed value (e.g., $\phi$ gets a finite mass).

Although this possibility was briefly mentioned in Ref. 2, we have recently come to see this as a truly distinct mode of graceful exit that evades some of the criticisms of earlier models. First, our recent numerical calculations suggest that viable models exist for a wide range of functional forms and parameters, significantly wider than Mode 1. (See Figs. 2 and 3 for some examples.) Second, the big bubble problem is easily avoided because few bubbles are nucleated $(\epsilon \ll 1)$ while the universe is inflating. (In Mode 1,the troublesome CMBR distortion comes from inflation of the many bubbles nucleated as $\epsilon$ gets close to unity. ${ }^{5}$ ) Almost the entire false vacuum is converted to true vacuum by bubbles nucleated after the expansion has become subluminal. These uninflated bubbles are infinitessimally smaller than the horizon at decoupling, much too small to distort the CMBR. There remains one criticism: that quartic interactions in any potential for $\phi$ must be suppressed by an amount comparable to the fine-tuned interactions in new or chaotic inflation. ${ }^{10}$ The fine-tuned interactions in new or chaotic inflation are essential elements that actually drive the inflationary expansion; they 
must be fine-tuned into a tiny range that must not include zero. Here, we would emphasize that the quartic interactions in $\phi$ play no role in inflation, and, hence, it is acceptable if symmetries prohibit them altogether. (Such symmetries exist in superstring models, for example.) Hence, it is difficult to judge how serious this last criticism is to be taken.

We turn, then, to the density perturbation spectrum generated by this novel mechanism for terminating inflation. According to the recent COBE results, ${ }^{11}$ the CMBR anisotropy can be fit to a scale-free power spectrum, $P(k) \propto k^{n}$, where $n=1.1 \pm 0.6$ ( $1 \sigma$ limit). Exponent $n=1$ corresponds to an exactly scale-invariant, Harrison-Zeldovich spectrum. New and chaotic inflation result in a nearly scale-invariant spectrum with logarithmic deviations $(\propto \log \lambda)$. We have computed the exponent $n$ by fitting the density perturbation spectrum to a scale-free form over astrophysical scales, $1-10^{4} \mathrm{Mpc}$. For new or chaotic inflation, our fit gives $n \approx 0.95$. For constant $\omega$, the density perturbation spectrum in Eq. (3) converts to a power spectrum with

$$
n=\frac{2 \omega-9}{2 \omega-1} \text {. }
$$

For large $\omega$ (approaching the limit of Einstein gravity), the spectrum is near $n=1$. As $\omega$ decreases, the spectrum shifts to smaller values of $n$, tipping the spectrum towards more power on large scales.

For Mode 1, we argued that $\omega$ must be less than 25 to avoid the big bubble problem. According to Eq. (四), this requires $n<0.84$, a significant tilt away from scale-invariance.

For Mode 2, a greater range of $n$ is possible. In general, the amplitude $\left.\frac{\delta \rho}{\rho}\right|_{H}(\lambda)$ is $\approx H^{2} / \dot{\phi}$ evaluated as scale $\lambda$ is stretched beyond the horizon during inflation. In Mode 2, $\omega$, which controls the rate of inflation, varies rapidly during inflation, especially within the final e-foldings. In this case, there is no 
simple analytic expression for the amplitude; rather, $\phi, \omega, H$, and $\left.\frac{\delta \rho}{\rho}\right|_{H}$ have to be tracked by numerically solving the equations of motion. Our results are summarized in Figs. 2-4. To compare with COBE, ${ }^{11}$ we fit the upper curves $\left(1-10^{4} \mathrm{Mpc}\right)$ to a scale-free spectrum and determine $n$. Fluctuations on these scales were generated 50-60 e-foldings before the end of inflation and depend on $\omega$ during that period. ${ }^{5,7,8}$

We find three classes of behavior, depending on the functional form for the non-minimal coupling, $f(\phi)$ : (1) If $f(\phi)$ varies exponentially with $\phi$ (see Fig. 2), then $\omega$ remains very large until the last 10 e-foldings of inflation. Consequently, on scales $1-10^{4} \mathrm{Mpc}$, the spectrum is flat, $n \rightarrow 1$, virtually indistinguishable from new inflation. This regime was strictly disallowed in the original (Mode 1) graceful exit mechanism for extended inflation. (2) If $f(\phi)$ varies more slowly with $\phi$ (e.g., a polynomial), $\omega$ changes more gradually. Hence, $\omega$ is closer to $1 / 2$ during the last 50-60 e-foldings of inflation compared to (1), leading to a spectrum tipped towards $n<1$ on scales $1-10^{4}$ Mpc. However, $\omega$ cannot be too small and still satisfy all other inflationary constraints. ${ }^{5}$ We find that $1 \gtrsim n \gtrsim 0.5$ is spanned by a plausible range of parameters and polynomial forms. (Yet smaller values of $n$ are possible, in principle, but only if $f(\phi)$ is increasingly fine-tuned.) (3) Wild variations can be "designed" into the spectrum by special choices of $f(\phi)$. For example, $\omega(\phi) \equiv f / 2\left[f^{\prime}\right]^{2}$ blows up at any near-inflection point of $f(\phi)$. Consequently, sharp "dips" in the perturbation spectrum (see Fig. 4) are created as $\phi$ passes the near-inflection point. The dips are similar to features created in "designer inflation" models ${ }^{12,13}$ in new or chaotic inflation. By choosing parameters carefully, the dip can be made to lie within $1-10^{4} \mathrm{Mpc}$ scales (Fig. 4a), which is probably ruled out by COBE. If the dip is placed 
just beyond the horizon, the spectrum on $1-10^{4} \mathrm{Mpc}$ scales appears to be scale-free (no dips), tipped towards $n>1$. Values $1.3 \gtrsim n \geq 1.0$ can be obtained. See Fig. 4b. What should be emphasized, though, is that cases (1) and (2) occur for a robust set of functional forms and parameters, whereas case (3) only occurs for a very special choice of $f(\phi)$.

Our conclusion is that extended inflation, based on the mode in which expansion becomes subluminal before escape from the false vacuum, is viable and robust. The power spectrum for extended inflation is scale-free with a wider range of possible power indices than previously thought possible: $1.0 \geq$ $n \gtrsim 0.5$ for plausible models, tilted towards more power on large scales $(n=1$ is exact Harrison-Zel'dovich). Recently, it has been shown that extended inflation models with tilts $0.84 \gtrsim n \gtrsim 0.5$ have the additional property that gravitational waves, rather than density perturbations, are the dominant contribution to cosmic microwave background fluctuations on large angular scales. ${ }^{14}$ The range $n>1$ or strong deviations from a scale-free spectrum require unnatural functional forms and fine-tuning. The predicted range is within the present $1 \sigma$-limit for COBE. ${ }^{11}$ However, future experiments, including analysis of years two or more from COBE, should improve the measurements and provide useful new constraints on inflation models. 


\section{References}

[1] D. La and P. J. Steinhardt, Phys. Rev. Lett. 62, 376 (1989).

[2] P. J. Steinhardt and F. S. Accetta, Phys. Rev. Lett. 64, 2740 (1990).

[3] A. H. Guth, Phys. Rev. D23, 347, (1981).

[4] E. Weinberg, Phys. Rev. D40, 3950 (1989).

[5] D. La, P.J. Steinhardt, and E.W. Bertschinger, Phys. Lett. B231, 231 (1989).

[6] C. Brans and R.H.Dicke, Phys. Rev. 124, 925 (1961).

[7] M.S. Turner, D.S. Salopek and E.W. Kolb, Phys. Rev. D42, 3925 (1990).

[8] A.H. Guth and B. Jain, Phys. Rev. D45, 426 (1992).

[9] C.W. Will and P.J. Steinhardt, to appear.

[10] F.C. Adams, K. Freese, and A. H. Guth, Phys. Rev. D43, 965 (1991).

[11] G. F. Smoot, et al., submitted to Astro. J. Lett. (1992).

[12] H.M. Hodges, G.R. Blumenthal, L.A. Kofman and J.R. Primack, Nucl. Phys. B335, 197 (1990).

[13] D.S. Salopek, J.R. Bond, and J.M. Bardeen, Phys. Rev. D40, 1753 (1989).

[14] R.L. Davis, H.M. Hodges, G.F. Smoot, P.J. Steinhardt, M.S. Turner, FERMILAB-Pub-92/168-A (1992). 


\section{FIGURE CAPTIONS}

1. Typical behavior of $\omega(\phi)=f / 2\left[f^{\prime}\right]^{2}$ for polynomial or exponential $f(\phi)$. In Mode 2, extended inflation begins when $\omega \gg 1$ and ends when $\omega$ falls below $1 / 2$. Bubble nucleation is negligible until $\omega \ll 1 / 2$, too late for inflation to make many big bubbles.

2. $\delta \rho /\left.\rho\right|_{H}$ as wavelength $\lambda$ enters the horizon shown for all observable scales (lower), with blow-up showing large scales only (upper). Scale-invariance or, equivalently, a power spectrum with $n=1$, corresponds to a horizontal line. For exponential $f(\phi)$, [here, $f(\phi)=M^{2} \exp (.048 \phi / M)$, where $\phi \leq M$ initially and $M \gtrsim M_{F}$, the spectrum on large scales can be fit to $n \approx 1$ [here, $n=0.96$ ].

3. Same as Fig. 2 except for polynomial $f(\phi)$ [here, $f(\phi)=A\left(\phi^{2}+B \phi^{4} / M^{2}\right)$, where $A=0.0061$ and $B=0.0021]$. The best-fit to upper curve is $n=0.8$. Values $1.0 \geq n \gtrsim 0.5$ can be obtained by varying $A$ and $B$, but $B / A$ must be increasingly tuned for yet smaller $n$.

4. Same as Fig. 2 except $f(\phi)=A\left(3.125 \delta \phi^{2}-1.66 \phi^{3} / M^{2}+0.25 \phi^{4}\right)$ is designed to have an inflection point as $\delta \rightarrow 1$. Depending on parameters, a sharp dip may be created on astrophysical scales (lower and upper left; $A=0.2$ and $\delta=1.0001$ ); or, it can be placed just beyond the horizon $\lambda \approx 10^{4}$ Mpc (upper right; $A=0.059$ and $\delta=1.001$ ), which appears scalefree within the horizon with $n \approx 1.3$. 